\title{
The Rate of Strong Consistency of the Nearest Neighbor Density Estimator for Negatively Dependent Random Variables
}

\author{
Xiang Zeng and Qunying Wu \\ College of Science, Guilin University of Technology, Guilin 541004, China \\ Correspondence should be addressed to Qunying Wu, wqy666@glut.edu.cn
}

Received 8 October 2012; Accepted 23 October 2012

Academic Editors: A. Bellouquid, J. A. Ferreira, and C. Lu

Copyright (c) 2012 X. Zeng and Q. Wu. This is an open access article distributed under the Creative Commons Attribution License, which permits unrestricted use, distribution, and reproduction in any medium, provided the original work is properly cited.

Let $\left\{X_{n}: n \geq 1\right\}$ be a sequence of negatively dependent random variables. Based on $\left(X_{1}, \ldots, X_{n}\right)$, in this paper we investigate the rate of pointwise consistency and strong consistency of the nonparametric density estimator proposed by $\mathrm{Yu}$. We extend the correspondent results under the negatively dependent samples.

\section{Introduction and Lemmas}

In Yu [1] a very simple and useful nonparametric estimate of a density based on a sample on $\mathrm{R}$ has been defined. If $m_{n}$ is an integer, the nonparametric estimate $f_{n}(x)$ of $f(x)$ is defined by dividing $2 m_{n}$ by $n$ times the length of the smallest interval containing $x$ which consists of $2 m_{n}$ of the $n$ observations in which half of them lie on the left side of $x$ and half on the right side. Formally,

$$
f_{n}(x)= \begin{cases}\frac{2 m_{n}}{n\left(X_{\left(2 m_{n}+j\right)}-X_{(j+1)}\right)} & \text { if } x \in\left[X_{\left(m_{n}+j\right)}, X_{\left(m_{n}+j+1\right)}\right), \text { for } j=0,1, \ldots, n-2 m_{n}, \\ 0 & \text { if } x<X_{\left(m_{n}\right)} \text { or } x \geq X_{\left(n-m_{n}+1\right)}\end{cases}
$$

where $\left(X_{(1)}, \ldots, X_{(n)}\right)$ is the order statistic of $\left(X_{1}, \ldots, X_{n}\right)$. In Yu [1] It is showed that in the case of independent and identically distributed observations $f_{n}(x)$ converges to $f(x)$ in probability for almost all $x$ and converges to $f(x)$ with probability one for almost all $x$. Moreover, the strong uniform consistency of this estimator for $f$ uniformly continuous was also established. 
Since Bozorgnia et al. [2] studied sequences of negatively dependent random variables, many statisticians have investigated this topic with interest. A series of useful results have been established. One can refer to Amini and Bozorgnia [3] for complete convergence for negatively dependent random variables, Fakoor and Azarnoosh [4] for probability inequalities for sums of negatively dependent random variables, Liu and Zhang [5] for the consistency and asymptotic normality of nearest neighbor density estimator under $\beta$-mixing condition, Wu [6] for complete convergence for weighted sums of sequences of negatively dependent random variables, and Wu and Jiang [7] for the strong consistency of estimator in linear model for negatively dependent random samples. From (1.1), we investigate the rate of consistency of the negatively dependent samples.

The following set of definitions and Lemmas will be needed.

Definition 1.1. Random variables $X$ and $Y$ are said to be negatively dependent (ND) if

$$
P(X \leq x, Y \leq y) \leq P(X \leq x) P(Y \leq y)
$$

for all $x, y \in \mathrm{R}$. A collection of random variables is said to be pairwise negatively dependent (PND) if every pair of random variables in the collection satisfies (1.2).

It is important to note that (1.2) implies

$$
P(X>x, Y>y) \leq P(X>x) P(Y>y)
$$

for all $x, y \in \mathrm{R}$. Moreover, it follows that (1.3) implies (1.2), and hence, (1.2) and (1.3) are equivalent. Ebrahimi and Ghosh [8] showed that (1.2) and (1.3) are not equivalent for a collection of three or more random variables. Consequently, the following definition is needed to define sequences of negatively dependent random variables.

Definition 1.2 (see [2]). The random variables $X_{1}, \ldots, X_{n}$ are said to be negatively dependent (ND) if for all real $x_{1}, \ldots, x_{n}$

$$
\begin{aligned}
& P\left(\bigcap_{j=1}^{n}\left(X_{j} \leq x_{j}\right)\right) \leq \prod_{j=1}^{n} P\left(X_{j} \leq x_{j}\right), \\
& P\left(\bigcap_{j=1}^{n}\left(X_{j}>x_{j}\right)\right) \leq \prod_{j=1}^{n} P\left(X_{j}>x_{j}\right) .
\end{aligned}
$$

An infinite random sequence $\left\{X_{n}: n \geq 1\right\}$ is said to be ND if every finite subset $X_{1}, \ldots, X_{n}$ is ND.

Lemma 1.3 (see [2]). Let $X_{1}, \ldots, X_{n}$ be $N D$ random variables and let $f_{1}(x), \ldots, f_{n}(x)$ be a sequence of Borel functions which all are monotone increasing (or all are monotone decreasing), then $f_{1}\left(X_{1}\right), \ldots, f_{n}\left(X_{n}\right)$ are still $N D$ random variables. 
Lemma 1.4 (see [7]). Let $\left\{X_{n}: n \geq 1\right\}$ be a sequence of negatively dependent sequence with $\mathbb{E} X_{n}=$ $0,\left|X_{n}\right| \leq b_{n}$, a.s. $(n=1,2, \ldots), t>0$, and $t \cdot \max _{1 \leq i \leq n} b_{i} \leq 1$, then

$$
P\left(\left|\sum_{i=1}^{n} X_{i}\right|>\varepsilon\right) \leq 2 \exp \left\{-t \varepsilon+t^{2} \sum_{i=1}^{n} \mathbb{E} X_{i}^{2}\right\}, \quad \text { for any } \varepsilon>0 \text {. }
$$

In particular, writing $\sigma_{n}^{2}=n^{-1} \sum_{i=1}^{n} \operatorname{var}\left(X_{i}\right), t=\varepsilon /\left(2 \sigma_{n}^{2}+b_{n} \varepsilon\right)$, we get

$$
P\left(\frac{1}{n}\left|\sum_{i=1}^{n} X_{i}\right|>\varepsilon\right) \leq 2 \exp \left\{-\frac{n \varepsilon^{2}}{2\left(2 \sigma_{n}^{2}+b_{n} \varepsilon\right)}\right\}, \quad \text { for any } \varepsilon>0 \text {. }
$$

\section{Main Results}

Let $\left\{X_{n}: n \geq 1\right\}$ be a sequence of negatively dependent random variables with common distribution function $F(x)$ and density $f(x)$ and let $\left(X_{(1)}, \ldots, X_{(n)}\right)$ be the order statistic of $\left(X_{1}, \ldots, X_{n}\right)$.

In the case of independent observations, in order to estimate $f(x)$ from a sample $\left(X_{1}, \ldots, X_{n}\right)$ Yu [1] considered (1.1) and showed both the pointwise consistency and uniform consistency for $f_{n}(x)$. However, in many situations we are dealing with dependent variables, so we will extend these results for the rate of consistency of nearest neighbor density estimator.

Our theorems are formulated in a more general setting.

Theorem 2.1. Let $\left\{X_{n}: n \geq 1\right\}$ be a negatively dependent sequence with common distribution function $F(x)$, and $0<F(x)<1$. There exists a sequence of positive numbers $\left\{\tau_{n}: n \geq 1\right\},\left\{\tau_{n}: n \geq\right.$ $1\}$ which verifies jointly with $\left\{m_{n}: n \geq 1\right\}$ that

$$
m_{n} \longrightarrow \infty, \quad \tau_{n} \longrightarrow 0, \quad \frac{m_{n}}{n \tau_{n}} \longrightarrow 0, \quad \text { as } n \longrightarrow \infty
$$

If $f$ satisfies the local Lipschitz condition at $x$, and

$$
\frac{\tau_{n}^{2} m_{n}^{2}}{n} \longrightarrow \infty
$$

then

$$
\lim _{n \rightarrow \infty} P\left(\left|f_{n}(x)-f(x)\right|>\varepsilon \tau_{n}\right)=0 .
$$

Theorem 2.2. Under the conditions of Theorem 2.1, If (2.2) instead becomes

$$
\frac{\tau_{n}^{2} m_{n}^{2}}{n \ln n} \longrightarrow \infty
$$

then

$$
\left|f_{n}(x)-f(x)\right|=o\left(\tau_{n}\right), \quad \text { a.s. }
$$


Proof of Theorem 2.1. For any $\varepsilon>0$, we have

$$
\begin{aligned}
P\left(\left|f_{n}(x)-f(x)\right|>\varepsilon \tau_{n}\right) \leq & P\left(x<X_{\left(m_{n}\right)}\right)+P\left(x \geq X_{\left(n-m_{n}+1\right)}\right) \\
& +P\left(X_{\left(m_{n}\right)} \leq x<X_{\left(n-m_{n}+1\right)},\left|f_{n}(x)-f(x)\right|>\varepsilon \tau_{n}\right) \\
& \widehat{=} J_{n 1}+J_{n 2}+J_{n 3} .
\end{aligned}
$$

Let's begin with proving $\sum_{n=1}^{\infty} J_{n 3}<\infty$. Denote $A_{n}=\left\{X_{\left(m_{n}\right)} \leq x<X_{\left(n-m_{n}+1\right)}\right\}$. When $A_{n}$ is true, (1.1) implies that there exists $j(x), 0 \leq j(x) \leq n-m_{n}$, such that $x \in\left[X_{\left(m_{n}+j(x)\right)}, X_{\left(m_{n}+j(x)+1\right)}\right)$. Denote $X_{1}^{(x)}=X_{(j(x)+1)}$ and $X_{2}^{(x)}=X_{\left(2 m_{n}+j(x)\right)}$. Then, we get

$$
\begin{aligned}
J_{n 3} & \leq P\left(A_{n}, f_{n}(x)<f(x)-\varepsilon \tau_{n}\right)+P\left(A_{n}, f_{n}(x)>f(x)+\varepsilon \tau_{n}\right) \\
& \leq P\left(A_{n}, f_{n}(x)<f(x)-\frac{\varepsilon \tau_{n}}{2}\right)+P\left(A_{n}, f_{n}(x)>f(x)+\varepsilon \tau_{n}\right) \\
& \widehat{=} P_{n 1}+P_{n 2} .
\end{aligned}
$$

If $f(x) \leq \varepsilon \tau_{n}$, then $\left(A_{n}, f_{n}(x)<f(x)-\varepsilon \tau_{n}\right)$ is the impossible event, namely, $P\left(A_{n}, f_{n}(x)<f(x)-\varepsilon \tau_{n}\right)=0$. Herein below, we suppose that $f(x)>\varepsilon \tau_{n}$ for $P_{n 1}$. Using the previous denotions, we obtain that

$$
\begin{aligned}
P_{n 1} & =P\left(X_{2}^{(x)}-X_{1}^{(x)}>\frac{2 m_{n}}{n\left(f(x)-\varepsilon \tau_{n} / 2\right)}, A_{n}\right) \\
& \leq P\left(X_{2}^{(x)}-x>\frac{m_{n}}{n\left(f(x)-\varepsilon \tau_{n} / 2\right)}, A_{n}\right)+P\left(x-X_{1}^{(x)}>\frac{m_{n}}{n\left(f(x)-\varepsilon \tau_{n} / 2\right)}, A_{n}\right) \\
& \hat{=} P_{n 1}^{\prime}+P_{n 1}^{\prime \prime}, \\
P_{n 2} & =P\left(X_{2}^{(x)}-X_{1}^{(x)}<\frac{2 m_{n}}{n\left(f(x)+\varepsilon \tau_{n}\right)}, A_{n}\right) \\
& \leq P\left(X_{2}^{(x)}-x<\frac{m_{n}}{n\left(f(x)+\varepsilon \tau_{n}\right)}, A_{n}\right)+P\left(x-X_{1}^{(x)}<\frac{m_{n}}{n\left(f(x)+\varepsilon \tau_{n}\right)}, A_{n}\right) \\
& \hat{=} P_{n 2}^{\prime}+P_{n 2}^{\prime \prime} .
\end{aligned}
$$

Write

$$
\begin{gathered}
r_{n}=\frac{m_{n}}{n\left(f(x)-\varepsilon \tau_{n} / 2\right)} \\
r_{n}^{\prime}=\frac{m_{n}}{n\left(f(x)+\varepsilon \tau_{n}\right)} .
\end{gathered}
$$


Since $f$ satisfies the local Lipschitz condition of order one at $x$, there exist constants $M>0$ and $\delta>0$, such that $|f(t)-f(x)| \leq M|t-x|$ whenever $|t-x|<\delta$, that is,

$$
f(x)-M|t-x| \leq f(t) \leq f(x)+M|t-x| .
$$

From (2.1), $m_{n} / n \tau_{n} \rightarrow 0$ and $\tau_{n} \rightarrow 0$. Thus, when $n$ is sufficiently large, we have

$$
\begin{gathered}
r_{n}=\frac{m_{n}}{n\left(f(x)-\varepsilon \tau_{n} / 2\right)} \leq \frac{m_{n}}{n(f(x)-f(x) / 2)}=\frac{m_{n}}{(f(x) / 2) n \tau_{n}} \tau_{n}<\frac{\varepsilon \tau_{n}}{4 M}<\delta, \\
r_{n}^{\prime}=\frac{m_{n}}{n\left(f(x)+\varepsilon \tau_{n}\right)} \leq \frac{m_{n}}{n f(x)}=\frac{m_{n}}{n \tau_{n}} \frac{\tau_{n}}{f(x)}<\frac{\varepsilon \tau_{n}}{2 M}<\delta .
\end{gathered}
$$

By (2.10), (2.11), we have

$$
\begin{aligned}
\mathbb{E} I_{\left(x \leq X_{i}<x+r_{n}\right)} & =\int_{x}^{x+r_{n}} f(t) \mathrm{d} t \geq\left(f(x)-M r_{n}\right) r_{n}=\frac{f(x)-M r_{n}}{f(x)-\varepsilon \tau_{n} / 2} \cdot \frac{m_{n}}{n} \\
& \geq \frac{f(x)-\varepsilon \tau_{n} / 4}{f(x)-\varepsilon \tau_{n} / 2} \cdot \frac{m_{n}}{n}=\frac{4 f(x)-\varepsilon \tau_{n}}{4 f(x)-2 \varepsilon \tau_{n}} \cdot \frac{m_{n}}{n}, \\
\mathbb{E} I_{\left(x \leq X_{i}<x+r_{n}^{\prime}\right)} & =\int_{x}^{x+r_{n}^{\prime}} f(t) \mathrm{d} t \leq\left(f(x)+M r_{n}^{\prime}\right) r_{n}^{\prime}=\frac{f(x)+M r_{n}^{\prime}}{f(x)+\varepsilon \tau_{n}} \cdot \frac{m_{n}}{n} \\
& \leq \frac{f(x)+\varepsilon \tau_{n} / 2}{f(x)+\varepsilon \tau_{n}} \cdot \frac{m_{n}}{n}=\frac{2 f(x)+\varepsilon \tau_{n}}{2 f(x)+2 \varepsilon \tau_{n}} \cdot \frac{m_{n}}{n}, \\
\mathbb{E} I_{\left(x-r_{n} \leq X_{i}<x\right)} & =\int_{x-r_{n}}^{x} f(t) \mathrm{d} t \geq\left(f(x)-M r_{n}\right) r_{n} \\
& \geq \frac{4 f(x)-\varepsilon \tau_{n}}{4 f(x)-2 \varepsilon \tau_{n}} \cdot \frac{m_{n}}{n}, \\
\mathbb{E} I_{\left(x-r_{n}^{\prime} \leq X_{i}<x\right)} & =\int_{x-r_{n}^{\prime}}^{x} f(t) \mathrm{d} t \leq\left(f(x)+M r_{n}^{\prime}\right) r_{n}^{\prime} \\
& \leq \frac{2 f(x)+\varepsilon \tau_{n}}{2 f(x)+2 \varepsilon \tau_{n}} \cdot \frac{m_{n}}{n} .
\end{aligned}
$$

So when $n$ is sufficiently large, we have by (2.12) and (2.13)

$$
\begin{aligned}
P_{n 1}^{\prime} & =P\left(X_{2}^{(x)}-x>\frac{m_{n}}{n\left(f(x)-\varepsilon \tau_{n} / 2\right)}, A_{n}\right) \\
& \leq P\left(X_{2}^{(x)}-x>r_{n}\right) \\
& =P\left(\sum_{i=1}^{n} I_{\left(x \leq X_{i}<x+r_{n}\right)} \leq m_{n}\right)
\end{aligned}
$$




$$
\begin{aligned}
& \leq P\left(\sum_{i=1}^{n}\left(I_{\left(x \leq X_{i}<x+r_{n}\right)}-\mathbb{E} I_{\left(x \leq X_{i}<x+r_{n}\right)}\right) \leq m_{n}-n \frac{4 f(x)-\varepsilon \tau_{n}}{4 f(x)-2 \varepsilon \tau_{n}} \cdot \frac{m_{n}}{n}\right) \\
& =P\left(\sum_{i=1}^{n} \xi_{i} \leq m_{n} \frac{-\varepsilon \tau_{n}}{4 f(x)-2 \varepsilon \tau_{n}}<0\right) \\
& \leq P\left(\left|\sum_{i=1}^{n} \xi_{i}\right| \geq m_{n} \frac{\varepsilon \tau_{n}}{4 f(x)-2 \varepsilon \tau_{n}}\right) \\
& \leq P\left(\left|\sum_{i=1}^{n} \xi_{i}\right| \geq m_{n} \frac{\varepsilon \tau_{n}}{4 f(x)}\right),
\end{aligned}
$$

where $\xi_{i}=I_{\left(x \leq X_{i}<x+r_{n}\right)}-\mathbb{E} I_{\left(x \leq X_{i}<x+r_{n}\right)}$.

Consider

$$
\begin{aligned}
P_{n 2}^{\prime} & =P\left(X_{2}^{(x)}-x<\frac{m_{n}}{n\left(f(x)+\varepsilon \tau_{n}\right)}, A_{n}\right) \\
& \leq P\left(X_{2}^{(x)}-x<r_{n}^{\prime}\right) \\
& =P\left(\sum_{i=1}^{n} I_{\left(x \leq X_{i}<x+r_{n}^{\prime}\right)} \geq m_{n}\right) \\
& \leq P\left(\sum_{i=1}^{n}\left(I_{\left(x \leq X_{i}<x+r_{n}^{\prime}\right)}-\mathbb{E} I_{\left(x \leq X_{i}<x+r_{n}^{\prime}\right)}\right) \geq m_{n}-n \frac{2 f(x)+\varepsilon \tau_{n}}{2 f(x)+2 \varepsilon \tau_{n}} \cdot \frac{m_{n}}{n}\right) \\
& =P\left(\sum_{i=1}^{n} \xi_{i}^{\prime} \geq m_{n} \frac{\varepsilon \tau_{n}}{2 f(x)+2 \varepsilon \tau_{n}}\right) \\
& \leq P\left(\sum_{i=1}^{n} \xi_{i}^{\prime} \geq m_{n} \frac{\varepsilon \tau_{n}}{4 f(x)}\right) \\
& \leq P\left(\left|\sum_{i=1}^{n} \xi_{i}^{\prime}\right| \geq m_{n} \frac{\varepsilon \tau_{n}}{4 f(x)}\right),
\end{aligned}
$$

where $\xi_{i}^{\prime}=I_{\left(x \leq X_{i}<x+r_{n}^{\prime}\right)}-\mathbb{E} I_{\left(x \leq X_{i}<x+r_{n}^{\prime}\right)}$. Likewise, when $n$ is sufficiently large, we have by (2.14) and (2.15)

$$
\begin{aligned}
& P_{n 1}^{\prime \prime} \leq P\left(\left|\sum_{i=1}^{n} \eta_{i}\right| \geq m_{n} \frac{\varepsilon \tau_{n}}{4 f(x)}\right), \\
& P_{n 2}^{\prime \prime} \leq P\left(\left|\sum_{i=1}^{n} \eta_{i}^{\prime}\right| \geq m_{n} \frac{\varepsilon \tau_{n}}{4 f(x)}\right),
\end{aligned}
$$

where $\eta_{i}=I_{\left(x-r_{n} \leq X_{i}<x\right)}-\mathbb{E} I_{\left(x-r_{n} \leq X_{i}<x\right)}, \eta_{i}^{\prime}=I_{\left(x-r_{n}^{\prime} \leq X_{i}<x\right)}-\mathbb{E} I_{\left(x-r_{n}^{\prime} \leq X_{i}<x\right)}$. 
ISRN Applied Mathematics

From (2.16) and (2.18), we get

$$
P_{n 1} \leq P\left(\left|\sum_{i=1}^{n} \xi_{i}\right| \geq m_{n} \frac{\varepsilon \tau_{n}}{4 f(x)}\right)+P\left(\left|\sum_{i=1}^{n} \eta_{i}\right| \geq m_{n} \frac{\varepsilon \tau_{n}}{4 f(x)}\right) .
$$

From (2.17) and (2.19), we get

$$
P_{n 2} \leq P\left(\left|\sum_{i=1}^{n} \xi_{i}^{\prime}\right| \geq m_{n} \frac{\varepsilon \tau_{n}}{4 f(x)}\right)+P\left(\left|\sum_{i=1}^{n} \eta_{i}^{\prime}\right| \geq m_{n} \frac{\varepsilon \tau_{n}}{4 f(x)}\right) .
$$

Write $\zeta_{i}=I_{\left(-\infty<\mathrm{X}_{i}<x+r_{n}\right)}-\mathbb{E} I_{\left(-\infty<\mathrm{X}_{i}<x+r_{n}\right)}, \zeta_{i}^{\prime}=I_{\left(-\infty<\mathrm{X}_{i}<x+r_{n}^{\prime}\right)}-\mathbb{E} I_{\left(-\infty<\mathrm{X}_{i}<x+r_{n}^{\prime}\right)}, \beta_{i}=I_{\left(-\infty<\mathrm{X}_{i}<x\right)}-$ $\mathbb{E} I_{\left(-\infty<X_{i}<x\right)}$.

Then

$$
\begin{aligned}
P\left(\left|\sum_{i=1}^{n} \xi_{i}\right| \geq m_{n} \frac{\varepsilon \tau_{n}}{4 f(x)}\right) & =P\left(\left|\sum_{i=1}^{n} \zeta_{i}-\sum_{i=1}^{n} \beta_{i}\right| \geq m_{n} \frac{\varepsilon \tau_{n}}{4 f(x)}\right) \\
& \leq P\left(\left|\sum_{i=1}^{n} \zeta_{i}\right| \geq m_{n} \frac{\varepsilon \tau_{n}}{8 f(x)}\right)+P\left(\left|\sum_{i=1}^{n} \beta_{i}\right| \geq m_{n} \frac{\varepsilon \tau_{n}}{8 f(x)}\right) \\
& =P\left(\frac{1}{n}\left|\sum_{i=1}^{n} \zeta_{i}\right| \geq \frac{a m_{n} \tau_{n}}{n}\right)+P\left(\frac{1}{n}\left|\sum_{i=1}^{n} \beta_{i}\right| \geq \frac{a m_{n} \tau_{n}}{n}\right), \\
P\left(\left|\sum_{i=1}^{n} \xi_{i}^{\prime}\right| \geq m_{n} \frac{\varepsilon \tau_{n}}{4 f(x)}\right) & =P\left(\left|\sum_{i=1}^{n} \zeta_{i}^{\prime}-\sum_{i=1}^{n} \beta_{i}\right| \geq m_{n} \frac{\varepsilon \tau_{n}}{4 f(x)}\right) \\
& \leq P\left(\left|\sum_{i=1}^{n} \zeta_{i}^{\prime}\right| \geq m_{n} \frac{\varepsilon \tau_{n}}{8 f(x)}\right)+P\left(\left|\sum_{i=1}^{n} \beta_{i}\right| \geq m_{n} \frac{\varepsilon \tau_{n}}{8 f(x)}\right) \\
& =P\left(\frac{1}{n}\left|\sum_{i=1}^{n} \zeta_{i}^{\prime}\right| \geq \frac{a m_{n} \tau_{n}}{n}\right)+P\left(\frac{1}{n}\left|\sum_{i=1}^{n} \beta_{i}\right| \geq \frac{a m_{n} \tau_{n}}{n}\right),
\end{aligned}
$$

where $a=\varepsilon / 8 f(x)$.

Obviously, $\left\{\zeta_{i}\right\},\left\{\zeta_{i}^{\prime}\right\}$, and $\left\{\beta_{i}\right\}$ are monotonic function's. According to Lemma 1.3, $\left\{\zeta_{i}\right\},\left\{\zeta_{i}^{\prime}\right\}$, and $\left\{\beta_{i}\right\}$ are still ND sequences. We will apply Lemma 1.4 to $\left\{\zeta_{i}\right\},\left\{\zeta_{i}^{\prime}\right\}$, and $\left\{\beta_{i}\right\}$. For $\left\{\zeta_{i}\right\}$ and $\left\{\zeta_{i}^{\prime}\right\}$ we have $\mathbb{E}\left(\zeta_{i}\right)=0, \mathbb{E}\left(\zeta_{i}^{\prime}\right)=0,\left|\zeta_{i}\right|=\left|I_{\left(-\infty<X_{i}<x+r_{n}\right)}-\mathbb{E} I_{\left(-\infty<X_{i}<x+r_{n}\right)}\right| \leq$ $\left|I_{\left(-\infty<X_{i}<x+r_{n}\right)}\right|+\left|\mathbb{E} I_{\left(-\infty<X_{i}<x+r_{n}\right)}\right| \leq 2,\left|\zeta_{i}^{\prime}\right| \leq 2, n^{-1} \sum_{i=1}^{n} \operatorname{var}\left(\zeta_{i}\right)=\operatorname{var}\left(I_{\left(-\infty<X_{i}<x+r_{n}\right)}-\mathbb{E} I_{\left(-\infty<X_{i}<x+r_{n}\right)}\right) \leq$ $1, n^{-1} \sum_{i=1}^{n} \operatorname{var}\left(\zeta_{i}^{\prime}\right) \leq 1$. By (2.1), when $n$ is sufficiently large, such that $\tau_{n} m_{n} / n<1$, then we have

$$
\begin{gathered}
P\left(\frac{1}{n}\left|\sum_{i=1}^{n} \zeta_{i}\right| \geq \frac{a m_{n} \tau_{n}}{n}\right) \leq 2 \exp \left\{-\frac{n\left(a m_{n} \tau_{n} / n\right)^{2}}{2\left(2+2\left(a m_{n} \tau_{n} / n\right)\right)}\right\}=2 \exp \left\{-\frac{c m_{n}^{2} \tau_{n}^{2}}{n}\right\}, \\
P\left(\frac{1}{n}\left|\sum_{i=1}^{n} \zeta_{i}^{\prime}\right| \geq \frac{a m_{n} \tau_{n}}{n}\right) \leq 2 \exp \left\{-\frac{c m_{n}^{2} \tau_{n}^{2}}{n}\right\},
\end{gathered}
$$

where $c=a^{2} / 4(1+a)$. 
By (2.2) and (2.24), then we have

$$
P\left(\frac{1}{n}\left|\sum_{i=1}^{n} \zeta_{i}\right| \geq \frac{a m_{n} \tau_{n}}{n}\right) \longrightarrow 0 .
$$

By (2.2) and (2.25), then we have

$$
P\left(\frac{1}{n}\left|\sum_{i=1}^{n} \zeta_{i}^{\prime}\right| \geq \frac{a m_{n} \tau_{n}}{n}\right) \longrightarrow 0 .
$$

Similarly,

$$
P\left(\frac{1}{n}\left|\sum_{i=1}^{n} \beta_{i}\right| \geq \frac{a m_{n} \tau_{n}}{n}\right) \longrightarrow 0 .
$$

From (2.22), (2.26), and (2.28), we get $P\left(\left|\sum_{i=1}^{n} \xi_{i}\right| \geq m_{n}\left(\varepsilon \tau_{n} / 4 f(x)\right)\right) \rightarrow 0$. Similarly, $P\left(\left|\sum_{i=1}^{n} \eta_{i}\right| \geq m_{n}\left(\varepsilon \tau_{n} / 4 f(x)\right)\right) \rightarrow 0$. By (2.20), then $P_{n 1} \rightarrow 0$. From (2.23), (2.27), and (2.28), we get $P\left(\left|\sum_{i=1}^{n} \xi_{i}^{\prime}\right| \geq m_{n}\left(\varepsilon \tau_{n}\right) / 4 f(x)\right) \rightarrow 0$. Similarly, $P\left(\left|\sum_{i=1}^{n} \eta_{i}^{\prime}\right| \geq m_{n}\left(\varepsilon \tau_{n} / 4 f(x)\right)\right) \rightarrow 0$. By (2.21), then $P_{n 2} \rightarrow 0$. Hereby, we have proved that $J_{n 3} \rightarrow 0$. It remains to show that $J_{n 1} \rightarrow 0$ and $J_{n 2} \rightarrow 0$. Denote

$$
\beta_{i}^{\prime}=I_{\left(x<X_{i}<\infty\right)}-\mathbb{E} I_{\left(x<X_{i}<\infty\right)} .
$$

According to the conditions of Theorem 2.1, we can see $0<\mathbb{E} I_{\left(-\infty<X_{i}<x\right)}=P\left(X_{i}<x\right)=$ $F(x)<1, m_{n} / n \rightarrow 0$, as $n \rightarrow \infty$. Then, there exist positive constants $a_{1}$ and $a_{2}$ such that $\mathbb{E} I_{\left(-\infty<X_{i}<x\right)}-m_{n} / n>a_{1}$ and $\mathbb{E} I_{\left(x<X_{i}<\infty\right)}-m_{n} / n>a_{2}$. When $n$ is sufficiently large, we have

$$
\begin{aligned}
J_{n 1} & =P\left(x<X_{\left(m_{n}\right)}\right) \\
& =P\left(\sum_{i=1}^{n} I_{\left(-\infty<X_{i}<x\right)} \leq m_{n}\right) \\
& =P\left(\sum_{i=1}^{n} \beta_{i} \leq m_{n}-n \mathbb{E} I_{\left(-\infty<X_{i}<x\right)}<-a_{1} n\right) \\
& \leq P\left(\left|\frac{1}{n} \sum_{i=1}^{n} \beta_{i}\right|>a_{1}\right),
\end{aligned}
$$


ISRN Applied Mathematics

$$
\begin{aligned}
J_{n 2} & =P\left(x \geq X_{\left(n-m_{n}+1\right)}\right) \\
& =P\left(\sum_{i=1}^{n} I_{\left(x<X_{i}<\infty\right)} \leq m_{n}\right) \\
& =P\left(\sum_{i=1}^{n} \beta_{i}^{\prime} \leq m_{n}-n \mathbb{E} I_{\left(x<X_{i}<\infty\right)}<-a_{2} n\right) \\
& \leq P\left(\left|\frac{1}{n} \sum_{i=1}^{n} \beta_{i}^{\prime}\right|>a_{2}\right) .
\end{aligned}
$$

We will apply Lemma 1.4 to $\left\{\beta_{i}\right\}$ and $\left\{\beta_{i}^{\prime}\right\}$. For $\left\{\beta_{i}\right\}$, we have $\mathbb{E}\left(\beta_{i}\right)=0,\left|\beta_{i}\right|=\mid I_{\left(-\infty<X_{i}<x\right)}-$ $\mathbb{E} I_{\left(-\infty<X_{i}<x\right)}|\leq| I_{\left(-\infty<X_{i}<x\right)}|+| \mathbb{E} I_{\left(-\infty<X_{i}<x\right)} \mid \leq 2, \sigma_{n}^{2}=n^{-1} \sum_{i=1}^{n} \operatorname{var}\left(\beta_{i}\right)=\operatorname{var}\left(\beta_{i}\right)=\operatorname{var}\left(I_{\left(-\infty<X_{i}<x\right)}-\right.$ $\left.\mathbb{E} I_{\left(-\infty<X_{i}<x\right)}\right) \leq 1$. When $n$ is sufficiently large, then we have

$$
P\left(\left|\frac{1}{n} \sum_{i=1}^{n} \beta_{i}\right|>a_{1}\right) \leq 2 \exp \left\{-\frac{n a_{1}^{2}}{2\left(2 \sigma_{n}^{2}+2 a_{1}\right)}\right\} \leq 2 \exp \left\{-\frac{n a_{1}^{2}}{2\left(2+2 a_{1}\right)}\right\}=2 \exp \left\{-c^{\prime} n\right\} \rightarrow 0,
$$

where $c^{\prime}=a_{1}^{2} / 2\left(2+2 a_{1}\right)$. Similarly,

$$
P\left(\left|\frac{1}{n} \sum_{i=1}^{n} \beta_{i}^{\prime}\right|>a_{2}\right) \leq 2 \exp \left\{-\frac{n a_{2}^{2}}{2\left(2+2 a_{2}\right)}\right\} \longrightarrow 0
$$

From (2.31) and (2.32), we can get $J_{n 1} \rightarrow 0, J_{n 2} \rightarrow 0$. This completes the proof of Theorem 2.1.

Proof of Theorem 2.2. By (2.4), when $n$ is sufficiently large, we get $c \tau_{n}^{2} m_{n}^{2} / n>2 \ln n=\ln n^{2}$. From (2.24), then we have

$$
P\left(\frac{1}{n}\left|\sum_{i=1}^{n} \zeta_{i}\right| \geq \frac{a m_{n} \tau_{n}}{n}\right) \leq 2 \exp \left\{-\frac{c \tau_{n}^{2} m_{n}^{2}}{n}\right\} \leq 2 \exp \left(-\ln n^{2}\right)=\frac{2}{n^{2}} .
$$

Similarly,

$$
P\left(\frac{1}{n}\left|\sum_{i=1}^{n} \eta_{i}\right| \geq \frac{a m_{n} \tau_{n}}{n}\right) \leq \frac{2}{n^{2}}
$$

From (2.33) and (2.34), we get $\sum_{n=1}^{\infty} P_{n 1}<\infty$. For $P_{n 2}$, the proof follows in the same way. From (2.7), we can get $\sum_{n=1}^{\infty} J_{n 3}<\infty$. Similarly, by (2.30), we have $\sum_{n=1}^{\infty} J_{n 1}<\infty$ and $\sum_{n=1}^{\infty} J_{n 2}<\infty$. From (2.6), we can get

$$
\sum_{n=1}^{\infty} P\left(\left|f_{n}(x)-f(x)\right|>\varepsilon \tau_{n}\right)<\infty
$$


According to the Borel-Cantelli lemma, we have

$$
\left|f_{n}(x)-f(x)\right|=o\left(\tau_{n}\right) . \quad \text { a.s. }
$$

\section{Acknowledgments}

The author is very grateful to the referees and the editors for their valuable comments and helpful suggestions that improved the clarity and readability of the paper. This work is supported by the National Natural Science Foundation of China (11061012) and the Key Laboratory of Spatial Information and Geomatics (1103108-08).

\section{References}

[1] J. Yu, "Consistency of an alternative nearest neighbor probability density function estimator," Statistical Research Report 1993-6, University of Umeoa, 1986.

[2] A. Bozorgnia, R. F. Patterson, and R. L. Taylor, "Limit theorems for ND r.v.'s," Technical Report 93-119, University of Georgia, 1993.

[3] D. M. Amini and A. Bozorgnia, "Complete convergence for negatively dependent random variables," Journal of Applied Mathematics and Stochastic Analysis, vol. 16, no. 2, pp. 121-126, 2003.

[4] V. Fakoor and H. A. Azarnoosh, "Probability inequalities for sums of negatively dependent random variables," Pakistan Journal of Statistics, vol. 21, no. 3, pp. 257-264, 2005.

[5] Y. Liu and Y. Zhang, "The consistency and asymptotic normality of nearest neighbor density estimator under $\alpha$-mixing condition," Acta Mathematica Scientia Series B, vol. 30, no. 3, pp. 733-738, 2010.

[6] Q. Wu, "Complete convergence for weighted sums of sequences of negatively dependent random variables," Journal of Probability and Statistics, vol. 2011, Article ID 202015, 16 pages, 2011.

[7] Q. Wu and Y. Jiang, "The strong consistency of $M$ estimator in a linear model for negatively dependent random samples," Communications in Statistics: Theory and Methods, vol. 40, no. 3, pp. 467-491, 2011.

[8] N. Ebrahimi and M. Ghosh, "Multivariate negative dependence," Communications in Statistics A, vol. 10, no. 4, pp. 307-337, 1981. 


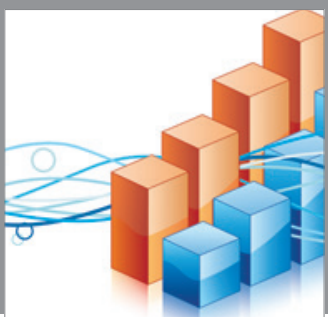

Advances in

Operations Research

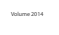

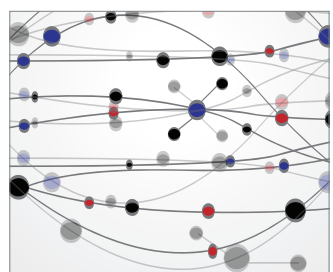

\section{The Scientific} World Journal
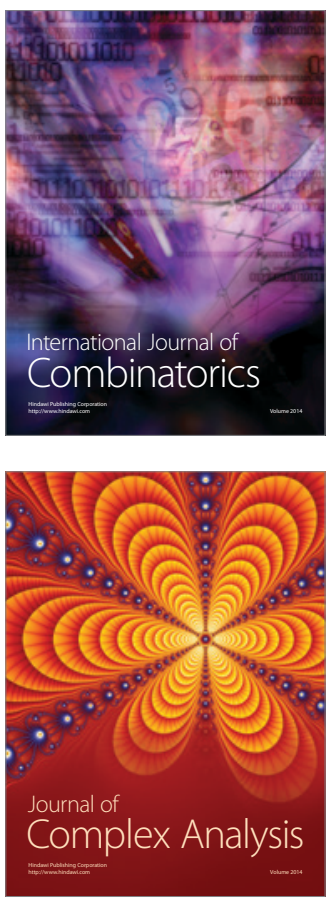

International Journal of

Mathematics and

Mathematical

Sciences
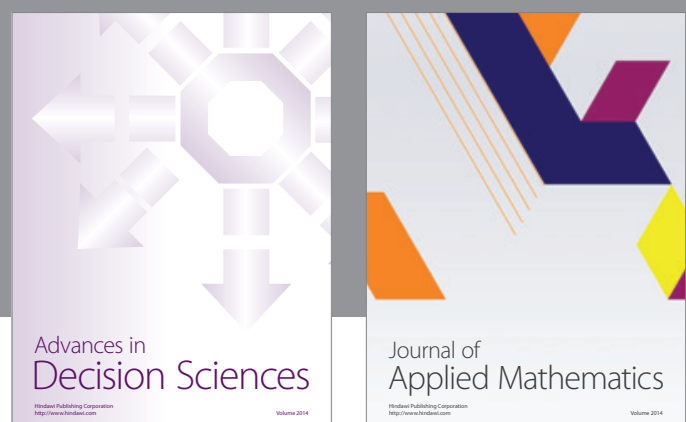

Journal of

Applied Mathematics
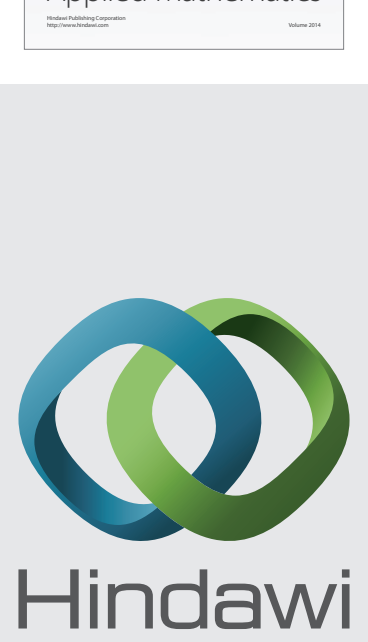

Submit your manuscripts at http://www.hindawi.com
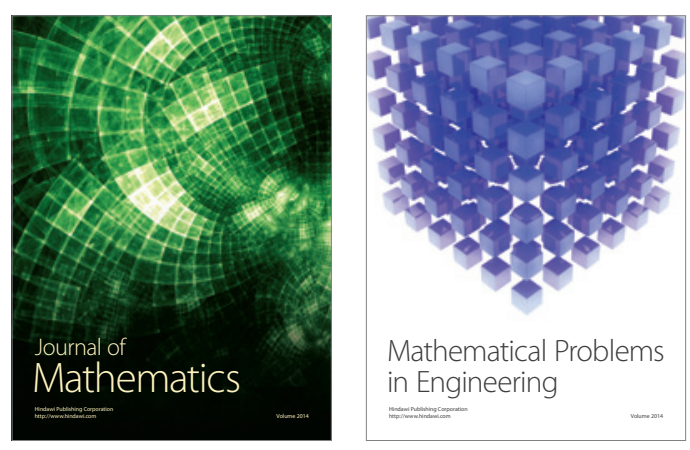

Mathematical Problems in Engineering
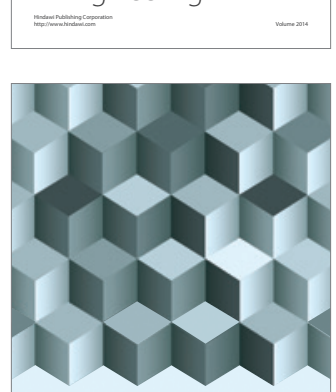

Journal of

Function Spaces
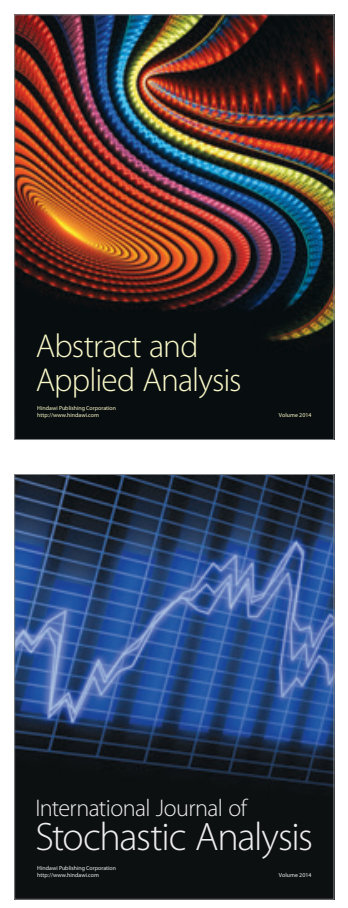

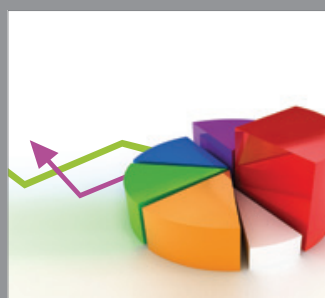

ournal of

Probability and Statistics

Promensencen
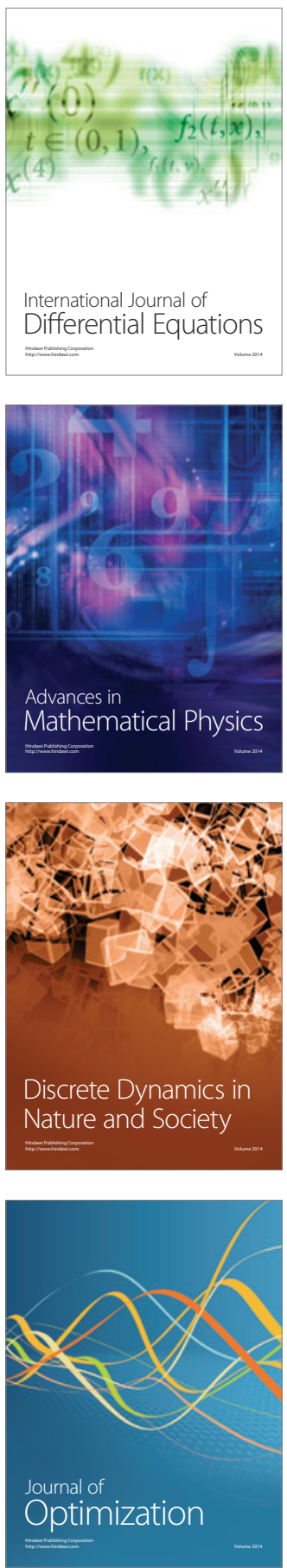